\title{
A Latent Class Extension of Signal Detection Theory, with Applications
}

\author{
Lawrence T. DeCarlo \\ Department of Human Development \\ Teachers College, Columbia University
}

A latent class extension of signal detection theory is presented and applications are illustrated. The approach is useful for situations where observers attempt to detect latent categorical events or where the goal of the analysis is to select or classify cases. Signal detection theory is shown to offer a simple summary of the observers' performance in terms of detection and response criteria. Implications of the view via signal detection for the training of raters are noted, as are approaches to validating the parameters and classifications. An extension of the signal detection model to more than two latent classes, with a simple restriction on the detection parameters, is introduced. Sample programs to fit the models using software for latent class analysis or software for second generation structural equation modeling are provided.

In many situations in psychology, education, and medicine, observers attempt to detect or discriminate between two or more classes of events. When the events are observable, the methods of signal detection theory (SDT) can be used to obtain a measure of an observer's ability to detect or discriminate (see Macmillan \& Creelman, 1991; Swets, 1996). Consider, for example, a simple test of recognition memory. A list of words can be presented during a study period and a combination of old words (from the list) and new words can be presented during a subsequent test, with the observers' task being to decide whether each word is old or new, or to rate their confidence that a word is old or new. From the perspective of SDT, the effect of an event (an old or new word) can be represented by a continuous latent variable, usually interpreted as an observer's perception of the event (e.g., the familiarity of the word), which is used together with a response criterion to arrive at a decision of old or new. The results can be summarized by a detection parameter, say $d$, which for the memory example is a measure of recognition memory strength, and by a parameter $c$ that indicates the location of the response criterion; the location of the criterion is viewed as

Correspondence concerning this article should be addressed to Lawrence T. DeCarlo, Department of Human Development, Box 118, Teachers College, Columbia University, 525 West $120^{\text {th }}$ Street, New York, NY 10027-6696. E-mail: decarlo@exchange.tc.columbia.edu 


\section{DeCarlo}

being largely arbitrary, in that it depends on motivational and other (usually uncontrolled) factors. Indeed, interest in SDT was stimulated in part by experiments in psychology that showed that although the response criteria of observers attempting to detect a signal varied considerably across different sessions (and could be experimentally manipulated), their detection remained approximately constant (e.g., Green \& Swets, 1988; Swets, Tanner, \& Birdsall, 1961). Since then, SDT has been shown to be quite useful for the study of cognitive and perceptual processes in psychology.

For the above example, it is known what type of event was presented on each trial, that is, whether an old or new word was presented. In other situations, the task is again one of signal detection, but the events are not observed. Examples are determining whether or not a person has a psychological or physical condition, such as depression or disease, whether an essay merits a pass or fail, or whether or not a slide shows a tumor. In these cases, the true state of the person or object (hereafter referred to simply as the case) is not known. As before, SDT assumes that the observer has a perception of the case that is used together with a criterion to arrive at a decision. Thus, the psychological theory is the same, the only difference is that the events of interest are not observed.

Signal detection theory can readily be applied to this type of situation by incorporating it into a latent class analysis. Latent class analysis (Clogg, 1995; Dayton, 1998; Hagenaars, 1993; McCutcheon, 1987) offers models that include unobserved (latent) classes or categories of persons or objects. Although signal detection models with latent classes have been used in medical applications (c.f. Henkelman, Kay, \& Bronskill, 1990; Quinn, 1989), their general applicability in psychology and education is not well known. The present article motivates the use of latent class SDT models and attempts to bridge some gaps between interrelated research in psychology, education, and medicine.

\section{Signal Detection Theory with Latent Classes}

Signal Detection Theory. Figure 1 illustrates the basic ideas of signal detection theory for the situation where there are two events (e.g., signal or noise, old or new word) and the observer uses a one to four rating response. It is assumed that the presentation of an event to an observer has an effect that can be represented by a random variable on an underlying continuum (e.g., the observer's perception). For two events, there are two probability distributions that differ in location, as shown in Figure 1. The distance between the distributions, denoted as $d$, reflects the observer's ability to discriminate between the two types of 


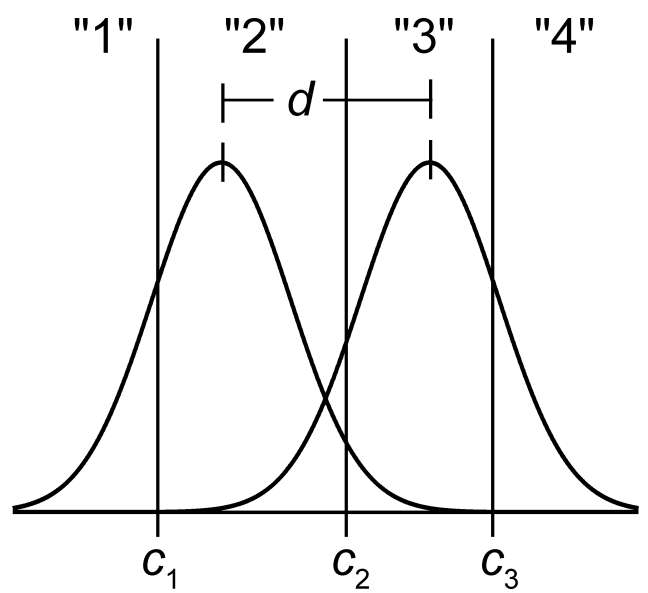

\section{Figure 1}

An Illustration of Signal Detection Theory

events. On each trial, the observer's perception of a case (the presented object) is a realization of a random variable that is used together with response criteria to arrive at an observed response. For example, as shown in Figure 1, the observer responds " 1 " if the realization is below the lowest criterion $\left(c_{1}\right)$, " 2 " if it is between the first and second criteria, and so on.

A general signal detection model for ordinal responses and two events that follows from the above assumptions can be written as

$$
p\left(Y_{i j} \leq k \mid X_{i}=x_{i}\right)=F\left[\left(c_{j k}-d_{j} x_{i}\right) / \tau_{j}\right]
$$

where $Y_{i j}$ is the response variable for observer $j(j=1, \ldots, J)$ to case $i$ $(i=1, \ldots, N), p\left(Y_{i j} \leq k \mid X_{i}=x_{i}\right)$ is the cumulative probability of response category $k$ (for $1 \leq k \leq K-1$ ) for observer $j$ given $X_{i}=x_{i}$, where $K$ is the number of response categories (which can vary across observers, and so should be written as $K_{j}$, but the situation with equal numbers of response categories across observers will be considered here to simplify notation) and $X_{i}$ is a dummy coded variable $\left(x_{i}=0,1\right)$ that indicates the presence or absence of an event for case $i,{ }^{1} c_{j k}$ is the $k^{\text {th }}$ response criterion for the $j^{\text {th }}$ observer with $c_{j 0}=-\infty, c_{j K}=\infty$ and $c_{j 1}<c_{j 2}<\ldots<c_{j, K-1}, d_{j}$ is the discrimination parameter for the $j^{\text {th }}$ observer, $F$ is a cumulative distribution function $(\mathrm{CDF})$, and $\tau_{j}$ is a scale parameter. The

\footnotetext{
${ }^{1}$ Using effect coding gives a re-parameterization where the coefficient of $x_{i}$ is $d_{j} / 2$ and the criteria are located with respect to the midpoint of the two distributions (see DeCarlo, 1998), denoted as $c_{j k}^{\prime}$ here.
} 


\section{DeCarlo}

logistic CDF is used for the examples presented here, so that the underlying distributions are logistic, however the model is more general in that other distributions can be used as well; some examples of SDT with normal and extreme value distributions are given in DeCarlo (1998). To use other distributions, Equation 1 can be re-written as a generalized linear model, in which case the choice of link function determines the form of the underlying distributions (see DeCarlo, 1998).

SDT with Latent Events. To extend the model to situations where the events are latent, the observed variable $X$ is replaced by a latent categorical variable, say $X^{*}$ with values $x_{c}^{*}=0,1$ for $c=1,2$,

$$
p\left(Y_{i j} \leq k \mid X^{*}=x_{c}^{*}\right)=F\left[\left(c_{j k}-d_{j} x_{c}^{*}\right) / \tau_{j}\right] .
$$

The model is the same as before, however the situation differs in that Equation 2 cannot be fit if there is only one observer (in contrast to Equation 1) because the model is not identified, that is, one cannot obtain unique estimates of the parameters. Several observers are necessary for the model to be identified; for example, for two latent classes, at least three observers are necessary if the responses are dichotomous, whereas at least two observers are necessary if the responses are ordinal with at least three categories. The models for the $J$ observers can then be incorporated into a latent class model, as shown next. Note that the scale parameter $\tau_{j}$ can be set to unity, without loss of generality, and this will be done from here on (the scale parameter has to be taken into account when comparing results from models with different link functions; see DeCarlo, 1998).

Latent Class Models. A discussion of latent class analysis is beyond the scope of the present article; the reader is referred to Dayton (1998) or Clogg (1995) for discussion and references. Here it is shown that the signal detection model given above is easily incorporated into a restricted latent class model, and some basic aspects of the analysis are illustrated.

Consider the situation where $J$ observers examine $N$ cases and make decisions in one of $K$ response categories. Note that, for each case, there is a pattern of responses (a vector) across the observers, which can be denoted as $\left(Y_{1}, Y_{2}, \ldots, Y_{J}\right)$. The total number of possible response patterns is $K^{J}$; for example, if three observers give ordinal ratings from one to four, then there are $4^{3}=64$ possible response patterns. Thus, the data for $J$ observers examining $N$ cases can be summarized by a multiway frequency table with $K^{J}$ cells, where each cell gives the number of cases with a particular response pattern. A latent class model can be viewed as a 
probability model for the response patterns. In particular, it is assumed that there are $C$ mutually exclusive and exhaustive latent classes so that the probabilities of the response patterns can be obtained by summing over the latent classes,

$$
p\left(Y_{1}, Y_{2}, Y_{3}\right)=\sum_{c=1}^{C} p\left(Y_{1}, Y_{2}, Y_{3}, X^{*}\right)=\sum_{c=1}^{C} p\left(X^{*}\right) p\left(Y_{1}, Y_{2}, Y_{3} \mid X^{*}\right)
$$

(for $J=3$ ) where $p\left(X^{*}=x_{c}^{*}\right)$ is the size (mixing proportion) of latent class $c$ with $p\left(X^{*}\right)>0$ for all $c$ and $\Sigma_{c} p\left(X^{*}=x_{c}^{*}\right)=1$ for $c=1$ to $C, p\left(Y_{1}, Y_{2}, Y_{3}\right)$ is the probability of response pattern $\left(Y_{1}, Y_{2}, Y_{3}\right)$, and $p\left(Y_{1}, Y_{2}, Y_{3} \mid X^{*}\right)$ is the conditional probability of response pattern $\left(Y_{1}, Y_{2}, Y_{3}\right)$ given $X^{*}=x_{c}^{*}$. In addition, conditional on the latent class, responses are assumed to be independent, so that

$$
p\left(Y_{1}, Y_{2}, Y_{3} \mid X^{*}\right)=p\left(Y_{1} \mid X^{*}\right) p\left(Y_{2} \mid X^{*}\right) p\left(Y_{3} \mid X^{*}\right),
$$

where $p\left(Y_{j} \mid X^{*}\right)$ is the conditional probability of response $k$ for observer $j$ given $X^{*}$ and $\Sigma_{k} p\left(Y_{j}=k \mid X^{*}\right)=1$. Equation 4 is an assumption of conditional independence; it reflects a basic assumption of latent class analysis, which is that the $J$ response variables are independent given the latent class.

Latent Class Models and Signal Detection. Equations 3 and 4 are the basic equations for an unrestricted latent class model. For restricted latent class models, the conditional probabilities of Equation 4 are restricted in various ways. For example, to incorporate the signal detection model of Equation 2, differences between the cumulative probabilities are used,

$$
\begin{aligned}
p\left(Y_{j}=k \mid X^{*}=x_{c}^{*}\right)=F\left(c_{j k}-d_{j} x_{c}^{*}\right) & k=1 \\
p\left(Y_{j}=k \mid X^{*}=x_{c}^{*}\right)=F\left(c_{j k}-d_{j} x_{c}^{*}\right)-F\left(c_{j k-1}-d_{j} x_{c}^{*}\right) & 1<k<K . \\
p\left(Y_{j}=k \mid X^{*}=x_{c}^{*}\right)=1-F\left(c_{j k-1}-d_{j} x_{c}^{*}\right) & k=K
\end{aligned}
$$

The result is a general class of signal detection models with latent classes, which are useful for situations that can be conceptualized in terms of SDT, such as when observers attempt to detect or discriminate latent categorical events. The models can be fit with software for latent class analysis that allows one to restrict the conditional probabilities using models with cumulative link functions. For example, the software LEM (Vermunt, 1997a), which is 


\section{DeCarlo}

freely available on the Internet (http://www.uvt.nl/faculteiten/fsw/ organisatie/departementen/mto/software $2 . \mathrm{html}$ ) was used for the analyses presented here; a sample LEM program for the binary response example discussed below is given in Appendix A. LEM allows one to fit a variety of latent class and latent trait models to categorical data using maximum likelihood; a version of the EM algorithm is used (see Vermunt, 1997a). For details about estimation and the use of the EM algorithm to fit latent class models see Bartholomew and Knott (1999), Heinen (1996), McCutcheon, (1987), McLachlan and Peel (2000), or Vermunt (1997b). The models considered here can also be fit using software for (second generation) structural equation modeling, such as Version 2 of Mplus (Muthén \& Muthén, 1998); a sample Mplus program for the ordinal response example discussed below is given in Appendix A and some other examples are given in DeCarlo (2001).

\section{Examples}

Binary Responses. For binary responses, the model is simply a reparameterization of the unrestricted latent class model (Equations 3 and 4). The reparameterization is useful, however, in that it provides a different perspective on and insights into the data. An example is a study concerned with pleural thickening (from Walter \& Irwig, 1988) used by Dayton (1998) to illustrate the application of latent class models to medical diagnosis. Three observers examined 1692 chest X-rays and made a yes/no decision as to the presence or absence of thickening of the lung tissues. From the signal detection perspective, an observer has a perception of each X-ray that they use in conjunction with a response criterion to arrive at a decision. Of basic interest is the parameter $d_{j}$, which reflects the $j^{\text {th }}$ observer's ability to detect thickening.

Table 1

SDT Parameter Estimates for Latent Class Logistic SDT Model: Pleural Thickening Data

\begin{tabular}{lcccc}
\hline & $d_{j}$ & $S E$ & $c_{j 1}$ & $S E$ \\
\cline { 2 - 5 } Observer A & 5.69 & 0.48 & 4.60 & 0.35 \\
Observer B & 3.89 & 0.32 & 3.30 & 0.14 \\
Observer C & 5.69 & 0.48 & 4.51 & 0.34 \\
\hline
\end{tabular}

Note. $S E=$ standard error. 
Table 1 presents results for a fit of a latent class logistic signal detection model (the data are given by Dayton, 1998, p. 26); a sample LEM program is given in Appendix A and some notes on using LEM are given in Appendix B. First note that the estimates of $d_{j}$ are all large, significant, and suggest excellent detection. For the logistic SDT model, $d_{j}$ are simply log odds ratios, and so the estimate of 3.89 for Observer B (the smallest estimate) indicates that his or her odds of detecting thickening are $\exp (3.89)=49$ times higher for an event (thickening) than a non-event (no thickening), which is quite good, as was also noted by Dayton (1998). Note that another (equivalent) view of $d_{j}$ is that it provides a measure of the conditional precision of each observer (c.f., Clogg \& Manning, 1996; Mellenberg, 1996); for a correlationtype measure, $d_{j}$ can be rescaled to a zero-one range as follows

$$
Q_{j}=\frac{e^{d_{j}}-1}{e^{d_{j}}+1},
$$

which is simply Yule's $Q$ (see Clogg \& Manning, 1996). For the pleural thickening data, the values of $Q_{j}$ are $.99, .96$, and .99 for Observers A, B, and C, respectively, which again indicates high precision (i.e., good detection) for each observer.

The parameter estimates in Table 1 suggest that $d_{j}$ is equal for Observers $\mathrm{A}$ and $\mathrm{C}$. The first row of Table 2 presents a likelihood ratio $(L R)$ goodness of fit test for a model with this restriction (note that the unrestricted model has as many parameters as observations and so fits perfectly). The $L R$ statistic is not significant, and so the model is not rejected. In contrast, the

Table 2

Results for Restricted Latent Class Logistic SDT Model: Pleural Thickening $\underline{\text { Data }(N=1692)}$

\begin{tabular}{lrrrrr}
\hline & $L R$ & $d f$ & $p$ & AIC & BIC \\
\hline Equal $d_{j}(\mathrm{~A}, \mathrm{C})$ & $<0.01$ & 1 & .99 & 1794.29 & 1827.89 \\
Equal $d_{j}(\mathrm{~A}, \mathrm{~B}, \mathrm{C})$ & 15.51 & 2 & $<.01$ & 1807.79 & 1834.96 \\
Equal $d_{j}(\mathrm{~A}, \mathrm{C})$ equal $c_{j k}(\mathrm{~A}, \mathrm{C})$ & 0.13 & 2 & .94 & 1792.42 & 1819.59 \\
Equal $d_{j}(\mathrm{~A}, \mathrm{C})$ equal $c_{j k}(\mathrm{~A}, \mathrm{~B}, \mathrm{C})$ & 2.99 & 3 & .39 & 1793.27 & 1815.01 \\
Equal $d_{j}(\mathrm{~A}, \mathrm{C})$ equal $c_{j k}^{\prime}(\mathrm{A}, \mathrm{B}, \mathrm{C})$ & 3.06 & 3 & .38 & 1793.35 & 1815.08 \\
\hline
\end{tabular}

Note. $L R=$ likelihood ratio goodness of fit statistic, BIC = Bayesian information criterion, $\mathrm{AIC}=$ Akaike's information criterion. 


\section{DeCarlo}

second row of Table 2 shows that a model with a restriction of equal $d_{j}$ across all three observers is rejected. Thus, the results suggest that Observers A and $\mathrm{C}$ detected equally well, whereas detection for Observer B was lower, although his or her detection was still quite good.

Further restrictions that can be considered are with respect to the response criteria, although these are usually viewed in SDT as being arbitrary (and so not of substantive interest). Here I simply note that some of the submodels considered by Dayton (1998) have a straightforward interpretation in terms of SDT. For example, the third row of Table 2 shows that a model with both detection and criteria restricted to be equal across Observers $\mathrm{A}$ and $\mathrm{C}$ is not rejected; this corresponds to submodel III of Dayton (see his Table 3.2, p.28; note that the results obtained with LEM exactly match those shown in Dayton's table). The fourth row shows that a model with detection equal across $\mathrm{A}$ and $\mathrm{C}$ and the criterion equal across all three observers is not rejected; this corresponds to submodel V of Dayton. Note, however, that there are different criterion measures that have been considered in SDT, and tests concerned with the criterion can give different results, depending on which measure is used. For example, the distance of the criterion from the mode of the lower distribution is used here (which distribution is lower can be determined by the output; see Appendix B), however an alternative measure locates the criterion with respect to its distance from the midpoint of the two (symmetric) distributions (e.g., as done in the choice theory parameterization of SDT; see DeCarlo, 1998; Macmillan \& Creelman, 1991). The last row of Table 2 shows a model with the criterion equal across the three observers for this alternative measure, denoted as $c_{j k}^{\prime}$ (where for symmetric distributions $c_{j k}^{\prime}=c_{j k}-1 / 2 d_{j}$ ). In this case, the $L R$ goodness of fit statistic differs only slightly from before and the model is not rejected (thus one cannot distinguish between alternate hypotheses about the criterion in this case). Nevertheless, it is important to keep in mind that the results of tests concerned with the criterion can differ for the different measures.

Table 2 also shows information criteria, and in particular Akaike's information criterion (AIC) and the Bayesian information criterion (BIC; see Burnham \& Anderson, 1998; Dayton, 1998; Lin \& Dayton, 1997), using versions based on the log-likelihood. The information criteria can be used to compare non-nested models, with smaller values indicating a better model; they can be used, for example, to compare models with different link functions, as shown below. Table 2 shows that AIC favors the model given in the third row whereas BIC favors the models given in the last two rows (c.f., Dayton, 1998, who also noted differences between AIC and BIC for this example). From the perspective of SDT, these models only differ with respect to whether Observer $\mathrm{B}$ had the same criterion location as the other observers, whereas both models are consistent with the conclusion that Observers $\mathrm{A}$ and $\mathrm{C}$ had equal detection. 
Finally, the estimates of the latent class size [i.e., $p\left(X^{*}\right)$ in Equation 3] are .95 for the lower latent class and .05 for the higher class; note that Dayton (1998) obtained the same values for the (different) models he considered. Thus, it is estimated that about $5 \%$ of the 1692 cases had pleural thickening.

In summary, a fit of the latent class signal detection model offers a simple interpretation of the data. The analysis suggests that all three observers detected well, with Observers $\mathrm{A}$ and $\mathrm{C}$ equal and Observer B lower. With respect to the response criterion, it is clarified that there are different types of restrictions across observers that can be examined; namely whether the criterion location is constant with respect to the lower distribution (in which case the observer is basically controlling the false alarm rate) or whether it is constant with respect to the midpoint of the two distributions; some implications of the different criterion measures have been considered in SDT (see Macmillan \& Creelman, 1991), but merit further study. It is also noted that tests of the criterion may be of little substantive interest, because of the arbitrary aspect of the criterion's location, but this depends on the particular research application.

Ordinal Responses. Further aspects of the approach via SDT are illustrated by an example with ordinal responses. In this case, examination of the estimated conditional probabilities, as done in latent class analysis (e.g., by Dayton, 1998, for the binary response example discussed above), may not be as informative as examination of the signal detection parameters.

The example is from a widely cited article by Landis and Koch (1977) where guidelines as to interpreting the magnitude of kappa (Cohen, 1960) were offered (i.e., $<0=$ poor, $.00-.20=$ slight, $.21-.40=$ fair, $.41-.60=$ moderate, $.61-.80=$ substantial, $.81-1.00=$ almost perfect, p.165; note that Fleiss, 1981, p. 218 , suggested $<.40$ as poor agreement, .40 to .75 as fair to good agreement, and $>.75$ as excellent agreement; in psychometrics, agreement less than .70 is generally considered poor). Two neurologists examined patient records and made a decision as to the presence or absence of multiple sclerosis; the data for 149 Winnipeg patients are examined here (the data are given in Landis \& Koch, 1977); for other analyses of these data, see Darroch and McCloud (1986) and Uebersax (1993b). Decisions with respect to the presence or absence of multiple sclerosis were made on a 1 to 4 scale, with $1=$ certain multiple sclerosis, 2 = probable multiple sclerosis, $3=$ possible multiple sclerosis, and 4 = doubtful, unlikely, or definitely not multiple sclerosis (the coding is reversed for the analysis presented here, so that higher numbers indicate a diagnosis of more probable multiple sclerosis). SDT assumes that the neurologists had a perception of symptoms for each patient, which they used together with response criteria to arrive at a response. 


\section{DeCarlo}

For the sample of 149 patients, kappa is .21, indicating slight agreement, according to the criteria suggested by Landis and Koch (1977) or poor agreement, by Fleiss' (1981) criteria; weighted kappa (Cohen, 1968) is .38, which also indicates poor agreement. Latent class SDT, on the other hand, offers additional information of interest, such as estimates of the observers' detection and response criteria, the sizes of the latent classes, and the accuracy of classifications based on the fitted model.

The upper half of Table 3 shows the parameter estimates and standard errors for a fit of a latent class logistic SDT model. It is apparent that the estimated detection parameters are close in magnitude across the two observers. Also note that some of the standard errors are large, which reflects a lack of precision with respect to estimation, or what Vermunt and Magidson (2000) refer to as "weak identification"; this is also indicated by the ratio of the largest to smallest eigenvalues of the information matrix (the eigenvalues are given in the LEM output), which in this case is $109.58 / 0.08=1370$, which is rather large. The lack of precision is due to the small sample size relative to the number of observers. That is, the precision of estimation depends on both the number of observers and on the sample size: with few observers, larger sample sizes are needed (for example, note that, in contrast to the current example, the ratio of eigenvalues for the binary response example discussed above with three observers and 1692 cases is 294.1/2.7 = 109),

Table 3

Parameter Estimates for Logistic Latent Class Signal Detection Model: Multiple Sclerosis Data

Unrestricted Model

\begin{tabular}{|c|c|c|c|c|c|c|c|c|}
\hline & $d_{j}$ & $S E$ & $c_{j 1}$ & $S E$ & $c_{j 2}$ & $S E$ & $c_{j 3}$ & $S E$ \\
\hline Neurologist A & 4.02 & 1.79 & -0.36 & 0.38 & 1.99 & 1.28 & 4.21 & 1.76 \\
\hline \multirow[t]{3}{*}{ Neurologist B } & 4.03 & 1.84 & -0.82 & 0.35 & -0.01 & 0.39 & 2.43 & 1.46 \\
\hline & \multicolumn{8}{|c|}{ Restricted Model (equal $d_{j}$ ) } \\
\hline & $d_{j}$ & $S E$ & $c_{j 1}$ & $S E$ & $c_{j 2}$ & $S E$ & $c_{j 3}$ & $S E$ \\
\hline Neurologist A & 4.02 & 0.45 & -0.36 & 0.34 & 1.99 & 0.55 & 4.22 & 0.51 \\
\hline Neurologist B & 4.02 & 0.45 & -0.82 & 0.34 & -0.01 & 0.35 & 2.43 & 0.55 \\
\hline
\end{tabular}

Note. $S E=$ standard error. 
whereas having more observers compensates for a small sample size ${ }^{2}$ a similar point has recently been made in the context of confirmatory factor analysis by Marsh, Hau, Balla, and Grayson (1998).

The lower half of Table 3 shows results for a model where the detection parameters are restricted to be equal across the two observers; the $L R$ goodness of fit statistic in this case is 11.27 on $7 d f(p=.13)$, and so the restricted model is not rejected. Note that several of the standard errors are considerably smaller; the ratio of largest to smallest eigenvalues in this case is $111.86 / 0.98=114$. Also note that the criteria estimates differ little, if at all, across the restricted and unrestricted models. A model with, in addition to detection, the response criteria restricted to be equal across both observers is rejected; the $L R$ goodness of fit statistic is 53.4 on $10 d f$ with $p<.01$ (a model with only the criteria restricted, and not $d_{j}$, is also rejected). ${ }^{3}$ Thus, the observers appear to detect equally, but their response criteria differ. Note that when the detection parameters are equal across observers, tests with the alternative criteria measure noted above lead to the same conclusions (since $c_{j k}^{\prime}=c_{j k}-1 / 2 d_{j}$ ).

In summary, a fit of the latent class SDT model suggests that the two neurologists detected well, and about equally. The latter conclusion, however, must be tempered by the fact that the detection parameters are not estimated precisely in this case, because of the small sample size relative to the number of observers (there might be insufficient power to detect differences in detection). The results also suggest that the response criteria differed across observers, and in particular the larger values of $c_{j 2}$ and $c_{j 3}$ for Neurologist A in Table 3 show that he or she had higher criteria for responses of 3 or 4 (probable or certain multiple sclerosis), and in this way Neurologist A was more conservative than Neurologist B with respect to a diagnosis of multiple sclerosis. Thus, SDT provides a simple summary of the performance of the observers, both with respect to detection $\left(d_{j}\right)$ and decision factors $\left(c_{j k}\right)$. In addition, SDT shows why agreement was poor, that is, the low agreement arose because of differences in the response criteria across the observers.

Classification. This section considers some additional results for the above example. The statistics noted here are the same as those used in ordinary latent class analysis, and so the reader is referred to Dayton

\footnotetext{
${ }^{2}$ Simulations I've conducted (manuscript in preparation), for example, suggest that, for a sample size of 100 , estimation is accurate with 10 observers.

${ }^{3}$ The models are nested and so differences between the log-likelihoods could be used to test the restricted models (using a likelihood ratio statistic). Using the difference test, the restricted models are rejected (as expected from the $L R$ goodness of fit statistics, which differ considerably in magnitude across the restricted and unrestricted models).
} 


\section{DeCarlo}

(1998) or Clogg (1995) for further discussion and references. The focus here is on the use of the statistics in the context of latent class signal detection analysis.

A practical goal is to classify the cases into one of the latent classes using the observed response patterns. This can be done using the posterior probability of $X^{*}$ for a given response pattern, which for three observers can be written as $p\left(X^{*} \mid Y_{1}, Y_{2}, Y_{3}\right)$. Note that, from Bayes' theorem,

$$
p\left(X^{*} \mid Y_{1}, Y_{2}, Y_{3}\right)=\frac{p\left(X^{*}\right) p\left(Y_{1}, Y_{2}, Y_{3} \mid X^{*}\right)}{\sum_{c=1}^{C} p\left(X^{*}\right) p\left(Y_{1}, Y_{2}, Y_{3} \mid X^{*}\right)},
$$

which shows that the posterior probabilities are determined by the latent class sizes, $p\left(X^{*}\right)$ and by $p\left(Y_{1}, Y_{2}, Y_{3} \mid X^{*}\right)$ the latter of which depends on the signal detection parameters, as shown by Equations 4 and 5. Thus, given estimates of the latent class sizes, the criteria, and the detection parameters, Equation 6 can be used to assign each case to the latent class for which its estimated posterior probability of class membership is highest (Goodman, 1974).

It is also of interest to measure the quality of the classifications. First, note that the estimated posterior probabilities can be used to obtain an estimate of the expected proportion of cases correctly classified (see Clogg, 1995; Dayton, 1998),

$$
P_{C}=\sum_{s}\left[n_{s} \times \max p\left(X^{*} \mid Y_{1}, Y_{2}, Y_{3}\right)\right] / N,
$$

(again illustrated for $J=3$ observers) where $s$ indicates each unique response pattern (e.g., there are $S=K^{J}$ unique patterns, as noted above), $n_{s}$ is the frequency of each pattern (i.e., number of cases with a particular pattern; note that either observed or estimated frequencies can be used, as noted by Clogg, 1995), $\max p\left(X^{*} \mid Y_{1}, Y_{2}, Y_{3}\right)$ is the maximum posterior probability (across the latent classes) for a given response pattern, and $N$ is the total number of cases. In words, if the maximum estimated posterior probability for a particular response pattern was, for example, .70 for Class 1 , then one would expect that classifying all cases with that response pattern into Class 1 would result in 70 percent of the cases being correctly classified. For the entire sample (and all the response patterns), the expected proportion correctly classified is then simply a weighted average of the maximum posterior probabilities for each pattern, as shown by Equation 7 . 
Next, note that if one simply classifies all the cases (i.e., the entire sample) into the latent class with the largest size, then one can expect to correctly classify that proportion of cases (i.e., by chance). A statistic that corrects for this is lambda, which can be written as

$$
\lambda=\frac{P_{C}-\max p\left(X^{*}\right)}{1-\max p\left(X^{*}\right)},
$$

Equation 8 shows that values of lambda greater than zero indicate that using the posterior probabilities to classify cases gives an increase in the proportion correctly classified over and above that obtained by simply assigning all cases to the latent class with the largest size. Another (equivalent) view of lambda is that it reflects the relative reduction in classification error (Clogg, 1995; Goodman \& Kruskal, 1954), where the classification error is simply one minus $P_{C}$.

With respect to the multiple sclerosis data, the estimated latent class sizes are .36 and .64 for Classes 1 and 2, respectively, with Class 2 representing the higher event, which can be interpreted as consisting of patients who possibly have multiple sclerosis. The estimate of the proportion correctly classified is .94 , and so the estimate of lambda is $(.94-.64) /(1-.64)=.83$, which means that there is a relative increase of $83 \%$ in the proportion correctly classified by using the observers' response patterns over simply classifying all cases into the latent class with the largest size. This suggests good classification, although it should be noted that there is an upward bias in the estimate of the proportion correctly classified, because estimation and classification are performed on the same set of data; to take this into account, Clogg (1995) suggested an approach based on multiple imputation (which merits further research).

In summary, the example shows that one can have good detection but poor agreement, in this case because the response criteria differed across the observers, and similarly, one can have good classification but poor agreement (classification depends on detection, the latent class sizes, and the number of observers). The latter point is relevant because in practice the goal is often to classify cases. Thus, it is important to determine if poor agreement arises because detection is poor, in which case classification might also be poor (this depends in part on how many observers there are), or if agreement is poor because of differences in the criteria across observers, as in the current example, in which case classification might be good (e.g., if detection is good). The relevant information is provided by the estimates of the detection and criteria parameters from the SDT analysis. 


\section{DeCarlo}

Some Notes on Rater Training. Here it is noted that the view via SDT also has implications for the training of raters. For example, in the context of performance assessment in education, Congdon and McQueen (2000) noted that "Presumably, the rater training which is a common feature of rating programs is in part intended to maximize inter-rater agreement. However, even extensive training has little effect on the standards maintained by raters..." (p. 164). In light of SDT, agreement depends on two aspects of the observers' performance, namely their use of response criteria and their ability to detect. With respect to classification, however, detection is of primary importance. To illustrate, Figure 2 shows the effects of detection and response criteria for a model with two latent classes (with sizes of .4 and .6 for the lower and higher classes, respectively) and three observers giving binary responses. In particular, the figure shows the proportion correctly classified for a latent class logistic SDT model where either the detection or criterion for one observer was varied and those for the other two observers remained constant. Of course, the relation between the observers' parameters and the proportion
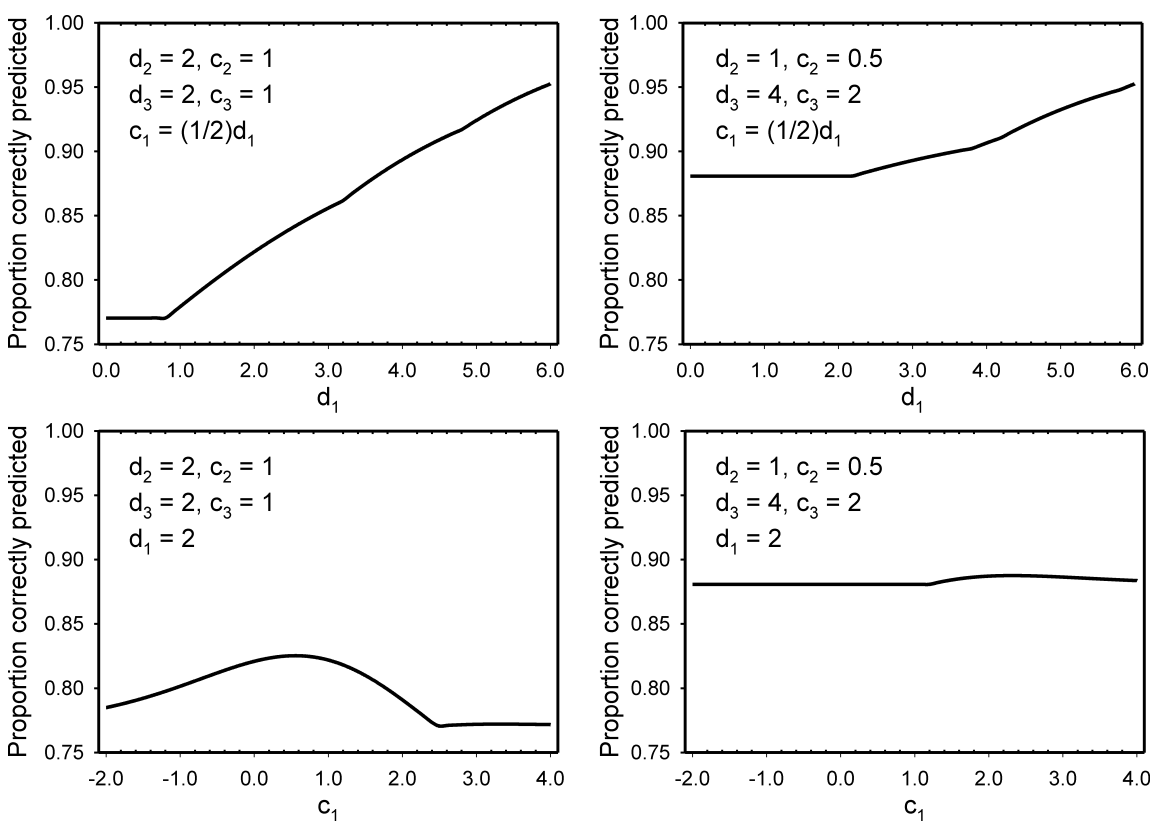

\section{Figure 2}

The top panels show, for a logistic SDT model with binary responses, the effect on the proportion correctly classified of varying detection for one observer, with the parameters for two other observers remaining constant; the bottom panels show the effect of varying the criterion for one observer, with the parameters for two other observers remaining constant. 
correctly classified is more complex (a variety of situations where the parameters were varied in different combinations were examined), but Figure 2 is representative of the results.

The top and bottom left panels of Figure 2 show situations where detection is moderate for two observers $\left(d_{2}\right.$ and $\left.d_{3}=2, Q=.76\right)$ and the criteria are located midway between the two distributions (other locations were examined as well). The top left panel shows that the proportion correctly classified increases nearly linearly as detection for one observer increases from zero to six. The bottom left panel shows that, with detection for all three observers fixed at two, varying the location of the criterion for one observer has only a small effect on the proportion correctly classified. The top and bottom right panels show a situation where detection for one observer is poor (i.e., $d_{2}=1, Q=.46$ ), detection for a second observer is $\operatorname{good}\left(d_{3}=4, Q=.91\right)$, and the criteria are again located at the midpoints. The top right panel shows that when detection $\left(d_{1}\right)$ is low, the proportion correctly classified is higher than that in the top left panel, which suggests that good detection for even one observer $\left(d_{3}=4\right)$ out of three has a large effect on the proportion correctly classified. Second, the panel shows that the proportion correctly classified increases when $d_{1}$ is greater than about 2 . In contrast, the bottom right panel shows that varying the location of the observer's criterion through a wide range has virtually no effect on the proportion correctly classified.

In short, Figure 2 illustrates that the best way to increase classification accuracy for a given set of observers is by increasing detection. ${ }^{4}$ As noted above, rater training tends to emphasize agreement (Congdon \& McQueen, 2000; Quellmalz, 1985), which from the perspective of SDT involves in part an attempt to get raters to use similar response criteria; research has shown, however, that this is difficult to do, even with extensive training. Moreover, because the criteria have little effect on classification accuracy, the focus on agreement is in some ways misdirected. Rather, the primary goal should be to improve observers' detection, since this has the largest effect on classification accuracy; the latent class SDT model might be useful in this regards, in that it allows one to examine the influence of a factor (e.g., training, instructions, practice, etc.) separately in terms of its effects on detection and/ or the response criteria. With respect to response criteria, the main goal should be to ensure that observers do not locate them far out in the decision space, which would result in little or no use of a response category (which can lead to estimation problems); in signal detection research, for example, observers are often instructed to try to use all of the response categories.

\footnotetext{
${ }^{4}$ Classification accuracy is also increased by using more observers (with nonzero $d_{j}$ ); it is important to consider in the design phase of a study how many observers it is practical to use.
} 


\section{DeCarlo}

\section{Validation}

As with any model, issues arise as to the validity of the model, its interpretation, and its application within a particular substantive context. Two basic aspects that should be assessed are the validity of the classifications and the validity of the SDT parameters.

Assessing Classification. This aspect of validation is the same as for ordinary latent class analysis, and so will only be briefly touched upon; examples of validation in latent class analysis can be found in Aitkin, Anderson, and Hinde (1981), Dayton (1998), and in chapters in Rost and Langeheine (1997). In some situations, information about the true states of cases might be available or might become available at a later time (e.g., in medicine or psychology); the known cases can then be used to validate the classifications. In other situations, known cases might not be available, but some other type of information might be available that can be used to validate the classifications (e.g., criterion validity). For example, in educational applications such as writing assessment, grades on exams or grade point average are often used as criteria in this way.

An issue that arises when known cases are not available is whether the latent classes correspond exactly to true categories. Uebersax (1988) noted, for example, that assuming an identity between the latent classes and true categories "is not implausible in many cases" (p. 409), but this has been a topic of some debate; for a discussion of this and other issues in the context of latent class analysis and item response theory, see Uebersax (1988) and the references therein. Here it is noted that, for a particular application, the identity issue should be considered, and, as part of the study design, one should consider what type of evidence as to validity can be obtained (e.g., criterion variables).

Assessing SDT Parameters. With respect to assessing the signal detection parameters, a basic question of interest is whether or not the estimated $d_{j}$ actually reflect the observers' ability to discriminate between latent events. This can be assessed in several ways, depending on whether or not cases with known statuses are available. If they are, then one approach would be to use the known cases in another session with the same set of observers. The estimates of $d_{j}$ could then be compared to those obtained in a session using unknown cases (note that the data from both sessions could be fit simultaneously); if the values were similar across the sessions (or perhaps just had a similar rank ordering), then that would suggest that the $d_{j}$ reflect some basic aspect of the observers, such as their ability to discriminate. 
The availability of known cases also offers other interesting possibilities. For example, estimation might be improved if one can include a proportion of known cases in with the unknown cases in the rating session; this is discussed as partial classification in mixture analysis (McLachlan \& Peel, 2000) or as training data in the Mplus user's guide (Muthén \& Muthén, 1998); note that a latent class signal detection model with partial classification can be fit using Mplus or LEM.

The situation is more complex in the absence of known cases. First, it is useful to note that a similar problem exists in item response theory and in confirmatory factor analysis; validating $d$, for example, is analogous to validating the discrimination parameter in item response theory or the factor loadings in confirmatory factor analysis. An approach similar to that used in those areas can be applied. For example, it would be of basic interest to determine if detection was invariant for a given set of observers over repeated sessions; similar values of $d_{j}$ across sessions would provide evidence that $d_{j}$ measures a stable characteristic. This is analogous to research in item response theory that has studied the invariance of item parameters across different groups of examinees (see Hambleton \& Swaminathan, 1985; Lord, 1980) or over time (e.g., Hoskens \& Wilson, 2001), and to research in confirmatory factor analysis that has studied the invariance of factor loadings across groups or time (e.g., Alwin \& Jackson, 1981; Byrne, Shavelson, \& Muthén, 1989). Note that, from the view via SDT, one would expect that discrimination might be invariant, but not the criteria (so there is only a partial invariance); also note that conclusions with respect to the criteria can differ, depending on which criteria measure is used, as discussed previously.

A useful aspect of the view via SDT is that it also suggests new experiments. For example, it would be interesting to try to induce observers to vary their criteria across different sessions, say by varying the instructions, and to see the effect, if any, on the SDT parameters, which is analogous to earlier research in experimental psychology with known cases; this could provide evidence for the validity of the criteria (e.g., if they can be systematically affected by instructions) and/or the detection parameter (e.g., if it is stable as the criteria vary). One could also try to experimentally manipulate discrimination, perhaps by varying factors such as amount of practice. Another possibility would be to use a contrasted-groups approach. For example, instead of using expert raters as gold standards (i.e., error free), one can allow for error in their judgments and compare them to a group of non-experts; the model could be fit to the data of both groups simultaneously and the estimated discrimination parameters compared; higher values for the experts could be viewed as evidence that $d_{j}$ reflects an 


\section{DeCarlo}

observer's ability to discriminate. These and other possibilities remain to be explored in future research.

\section{Extensions}

The focus here has been on the utility of the two class signal detection model, but there are several ways the basic model can be extended. These can be classified as extensions with respect to the signal detection part of the model or with respect to the latent classes, such as increasing their number or including additional latent variables.

With regards to extensions of the signal detection model, note that although the focus here has been on the logistic model with ordinal responses, the model is actually more general in that different link functions can be used, and so a wide range of models can be considered. Examples of using different link functions with SDT models for observed events are given in DeCarlo (1998); an example with latent events is given below. Other possibilities are to allow the variances of the underlying distributions to differ across the latent classes, as in the unequal variance extension of the signal detection model for observed events (Green \& Swets, 1988), or to use a mixture extension of SDT (DeCarlo, 2002).

Other extensions are with respect to the latent classes. One option is to increase the number of latent classes. As discussed next, an interesting extension is to increase the number of latent classes, but with a restriction on the discrimination parameters.

An Equal Distance SDT Model. In the two class SDT model, the values for $x_{c}^{*}=0,1$ for $c=1,2$ and $p\left(X^{*}\right)$ is estimated. One extension uses more values for $X^{*}$ and in particular $x_{c}^{*}=0,1, \ldots, C-1$ for the $c=1$ to $C$ latent classes. Here I note that SDT provides a simple interpretation of the resulting model. The approach is similar to that used in log-linear association models, as discussed by Clogg (1988), with the difference that the later are formulated within a log-linear framework (i.e., the models use adjacent category logits, not cumulative links). The extended SDT model discussed here (with logit link) can be viewed as a latent class extension of the uniform association model for cumulative odds ratios discussed by Agresti (1990); it is also more general in that other links can be used.

Specifically, let the values of $X^{*}$ be $x_{c}^{*}=0,1, \ldots, C-1$ with $\mathrm{p}\left(X^{*}\right)$ free, as in the basic two class model (still with a sum to one constraint on the probabilities). This is tantamount to assuming that the $C$ underlying distributions are ordered and equally spaced within each observer. That is, Equation 2 shows that with this coding scheme, $d_{j 2}=2 d_{j 1}$, where $d_{j 1}$ is the 
distance of distribution 1 from the reference distribution for observer $j$ and $d_{j 2}$ is the distance to distribution $2, d_{j 3}=3 d_{j 1}$, and so on. Figure 3 provides an illustration of the model with three latent classes. The figure shows that there is an equal spacing constraint, and so the model will be referred here to as the equal distance SDT model. The model is a restricted version of a signal detection model with more than two latent classes, with the restriction that the spacing of the distributions is equal within observers, but varies across observers. Note that, although values are assigned to $X^{*}$, no assumptions are made with respect to spacing of the latent classes (i.e., the equal spacing is in the observers' perceptions, not the latent classes); it is only assumed that there are $C$ (ordered) latent classes with sizes $p\left(X^{*}\right)$, with a multinomial distribution used for $p\left(X^{*}\right)$. This suggests a simple yet useful generalization of the basic latent class SDT model.

Example: Equal Distance SDT. A widely analyzed data set (e.g., Dillan \& Mulani, 1984; Uebersax, 1993c; Vermunt \& Magidson, 2000) presented in Dillon and Mulani (1984; and in Vermunt \& Magidson, 2000) provides an interesting example. The task of three observers was to determine whether each of 164 individuals were positive, neutral, or negative towards an object. The approach via SDT assumes that the responses are ordered, with neutral

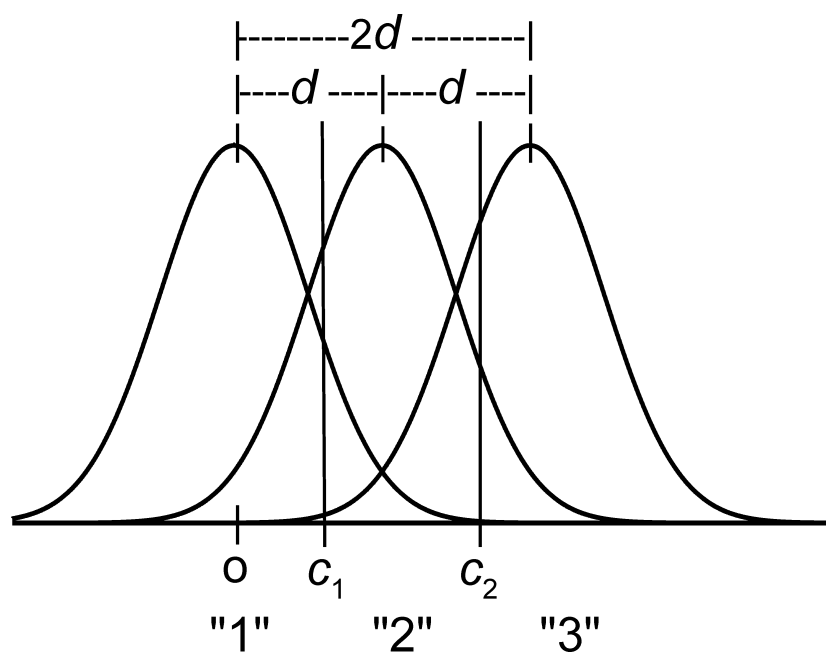

Figure 3

Latent Class Signal Detection Theory with Three Latent Classes and an Equal Distance Restriction 


\section{DeCarlo}

between positive and negative (responses were coded as $1=$ negative, $2=$ neutral, and $3=$ positive). Dillon and Mulani (1984) noted that an unrestricted latent class model with three classes provided a good fit of the data (several of the smallest eigenvalues are zero, however, which indicates an empirical identification problem; Dillon and Mulani noted that they added a constant to the table cells, which is not done here).

Table 4 presents goodness of fit statistics and information criteria for fits of several models with three latent classes; all the models were specified and fit using LEM (Vermunt, 1997a). In this case, both the likelihood ratio and chi-square statistics are shown, because they differ in some cases (whereas this wasn't the case for the examples presented above); this most likely occurs because there are many cells with small expected frequencies (i.e., there are many with expected frequencies less than five, and several with expected frequencies less than one), and so the goodness of fit statistics should be viewed with caution. For purposes of comparison, two models used as examples in the Latent Gold user's guide (Vermunt \& Magidson, 2000) for this data were also fit (using LEM) and the results are included in Table $4 ;{ }^{5}$ they are labeled as association models, because they are latent class extensions of row and uniform association models discussed by Agresti (1990), and are basically log-linear models with scores (also see Clogg \& Shihadeh, 1994). A basic difference between the SDT models and the

\section{Table 4}

Goodness of Fit Statistics and Information Criteria for Various Models, Equal Distance SDT Example

\begin{tabular}{lccccccc}
\hline & $d f$ & $X^{2}$ & $p$ & $L R$ & $p$ & AIC & BIC \\
\hline Association, 3 classes & 12 & 21.10 & 0.05 & 17.93 & 0.12 & 778.74 & 822.14 \\
Logit SDT, 3 classes & 12 & 19.99 & 0.07 & 17.28 & 0.14 & 778.09 & 821.49 \\
Association, 1 factor, 3 levels & 15 & 30.58 & 0.01 & 20.79 & 0.14 & 775.60 & 809.69 \\
Logit SDT, 3 equal distance & 15 & 25.76 & 0.04 & 19.31 & 0.20 & 774.12 & 808.22 \\
Log-log SDT, 3 equal distance & 15 & 16.47 & 0.35 & 16.98 & 0.32 & 771.79 & 805.89 \\
\hline
\end{tabular}

Note. $X^{2}=$ chi-square test, $L R=$ likelihood ratio test, $\mathrm{AIC}=$ Akaike's information criterion, $\mathrm{BIC}$ $=$ Bayesian information criterion.

\footnotetext{
${ }^{5}$ The results from LEM differ slightly from those given in the Latent Gold manual because of technical differences; for example, the latter uses Bayes constants. For Latent Gold, setting the Bayes constants to zero, the Newton-Raphson iterations to zero, and the convergence criteria to $0.1^{-8}$ gave identical or near identical results to LEM.
} 
association models is that the SDT models use cumulative links whereas the association models use adjacent category logits.

The first two models in Table 4 with 12 degrees of freedom are not nested and differ only with respect to the link function. The first is an association model that is equivalent to the ordinal indicator, three nominal cluster model discussed by Vermunt and Magidson (2000, see p.138), followed by a latent class logistic SDT model with three latent classes. The goodness of fit statistics suggest that the fit of both models is satisfactory. The next three models have $15 d f$; the first is an association model with one three level factor, which is referred to by Vermunt and Magidson (2000) as an ordinal three-level factor model with ordinal indicators. Next are two equal distance three class SDT models with logit and log-log links. Vermunt and Magidson (2000) noted that, of the models they considered, the BIC indicated that the association model with one three-level factor was the preferred model. Table 4 shows that both the AIC and BIC are smaller for the equal distance SDT models and are smallest for the model with log-log link (the log-log link implies that the underlying distributions are skewed to the right). Thus, the results suggest that the three class equal distance SDT models provide a parsimonious description of the data.

Table 5 presents parameter estimates for the latent class logistic SDT model with three classes (the logistic results are shown to allow comparison to the other examples presented above). The table shows that discrimination is excellent, with the distance between each of the underlying distributions being four or more. Although the estimate of $d_{j}$ for Observer B is higher than that for the other two observers (Vermunt \& Magidson, 2000, in essence concluded that this observer detected best), the standard errors are large, and a model with detection restricted to be equal across the three observers gives a goodness of fit statistic of $L R=20.12, d f=17, p=.27$. The estimates

Table 5

Parameter Estimates for Latent Class Logistic Equal Distance Signal Detection Model with Three Classes

\begin{tabular}{lcccccc}
\hline & $d_{j}$ & $S E$ & $c_{j 1}$ & $S E$ & $c_{j 2}$ & $S E$ \\
\hline Observer A & 4.06 & 0.56 & 2.23 & 0.59 & 6.33 & 0.94 \\
Observer B & 5.08 & 1.25 & 2.99 & 1.16 & 5.60 & 1.32 \\
Observer C & 4.07 & 0.52 & 1.66 & 0.48 & 5.74 & 0.80 \\
\hline
\end{tabular}

Note. $S E=$ standard error. 


\section{DeCarlo}

of the latent class sizes for the negative, neutral, and positive classes are .21, .36 , and .43 , respectively, whereas the estimate of $P_{C}=.95$ and $\lambda=.90$ (note that the latter estimate differs slightly from .91 , computed with the values reported here, because of rounding).

In summary, the results are concisely and meaningfully summarized in terms of the latent class equal distance SDT model: (a) detection is good, (b) the observers do not differ with respect to detection (or there is insufficient information to detect differences across observers), (c) the observers perceive the neutral class as being midway between the negative and positive classes, and (d) classification appears to be good. Note that a comparison of latent class models with cumulative link functions to association models merits further attention; the information criteria in Table 4, for example, suggest that in this case the (SDT) models with cumulative links are preferred over the (association) models with adjacent category logits, but the differences appear to be small (in this example, the models are near equivalent in terms of fit, but nevertheless are conceptually quite different).

Covariates. Another extension of the latent class SDT model is to include covariates, as has been done for latent class models (e.g., Dayton \& Macready, 1988; van der Heijden, Dessens, \& Bockenholt, 1996). The most general model allows for an effect of covariates on the latent class probabilities and also direct effects of covariates on the response probabilities. Although either or both of these types of effects can be included, attention must be given to the interpretation of the resulting model. For example, from the perspective of SDT, allowing the covariates to affect the response probabilities corresponds to a situation where the covariates are viewed as factors that affect the observers' perceptions (using covariates in this way is analogous to allowing for a type of differential item functioning in item response theory), whereas allowing them to affect the latent class probabilities corresponds to a situation where interest centers on using the covariates to predict latent class membership (e.g., to study group differences, such as across gender or ethnicity; this is analogous to multiple indicator, multiple cause models in structural equation modeling). Research on the use of covariates in latent class signal detection models is needed.

There are many other applications and extensions that can be considered. For example, the latent class SDT model presented here can also be used in situations where the observers rate only a portion of the cases, as done in designs with a large number of cases. There are then missing values (by design) for each observer (i.e., the cases the observer did not rate). The values are missing completely at random (see Little \& Rubin, 1987) and the latent class SDT model can be fit (using LEM, for example) by analyzing subtables 
(with appropriate parameter restrictions across tables) and including an indicator variable for the missing values, as discussed by Vermunt (1997a; 1997b). Another extension is to relax the assumption of conditional independence by, for example, introducing random effects (e.g., Qu, Tan, \& Kutner, 1996; Uebersax, 1999). One can also consider multidimensional models with additional categorical or continuous latent variables. These remain to be explored in future research. Some issues and limitations should also be noted: larger sample sizes or more observers might be needed for adequate estimation with more complex models, for example, issues of equivalent or near equivalent models (that possibly lead to different conclusions) arise, and the need for validation is as important as ever.

Comments on Some Related Models. The latent class SDT model is closely related to located latent class models (Uebersax, 1993b, 1993c) and to a latent trait finite mixture model discussed by Uebersax (1993a). Using Uebersax's (1993c) notation (and the subscripts used here), a located latent class model for ordinal responses can be written as

$$
p\left(Y_{i j} \leq k \mid \alpha_{j}, \tau_{j}, B_{c}\right)=\frac{1}{1+\exp \left[-1.7 \alpha_{j}\left(\tau_{j k}-B_{c}\right)\right]} .
$$

For two classes, $c=1,2$, the above is equivalent to the two class SDT model with a logit link and the following relations between the SDT parameters and the located latent class parameters: $c_{j k}=\tau_{j k}, d_{j}=1, \tau_{j}=1 /\left(1.7 \alpha_{j}\right), B_{1}=0$, and $B_{2}=1$. Uebersax (1993c) refers to $\alpha_{j}$ as a measurement error parameter; the above shows that it is equivalent to the inverse of the scale parameter $\tau_{j}$ in the SDT model. Note that, because $d_{j}$ is restricted to be unity in the located latent class model, $\alpha_{j}$ plays the same role as the detection parameter in the latent class signal detection model; this simply reflects the arbitrary scale: in SDT, $d_{j}$ is the distance between the underlying distributions with the variance fixed, whereas in located latent class analysis, the distance $B_{2}-B_{1}$ is fixed and $\alpha_{j}$ reflects the conditional precision (i.e., the variance of the underlying distributions). There are also differences in the approaches when applied to more than two latent classes, a discussion of which is outside the scope of the present article.

Another extension is to use a probability distribution for the latent classes, in which case the models are closely related to discretized versions of item response models (see Heinen, 1996). For example, using a discretized normal distribution for $p\left(X^{*}\right)$ and a logistic cumulative link function gives a model that is closely related to the graded response model 


\section{DeCarlo}

(Samejima, 1969). The conceptualization of the model differs, however, in that in SDT randomness arises from an observer's perception of a case whereas this is not the view with respect to item response theory. It would be informative to compare the different approaches in future research.

\section{Conclusion}

Signal detection theory with latent classes offers a simple and psychologically motivated approach to situations where observers make judgments about latent events. The view via SDT suggests that a large body of research and theory in psychology is relevant to models used in education, medicine, biostatistics, and psychometrics. There are many directions for future research, including experimental studies and comparisons and contrasts of different models.

\section{References}

Agresti, A. (1990). Categorical data analysis. New York: Wiley.

Aitkin, M., Anderson, D., \& Hinde, J. (1981). Statistical modelling of data on teaching styles. Journal of the Royal Statistical Society, A, 144, 419-448.

Alwin, D. F. \& Jackson, D. J. (1981). Applications of simultaneous factor analysis to issues of factorial invariance. In D. J. Jackson \& E. F. Borgatta (Eds.), Factor analysis and measurement in sociological research (pp. 249-279). Beverly Hills, CA: Sage.

Bartholomew, D. J. \& Knott, M. (1999). Latent variable models and factor analysis (2 ${ }^{\text {nd }}$ ed.). New York: Oxford University Press.

Burnham, K. P. \& Anderson, D. R. (1998). Model selection and inference: A practical information-theoretic approach. New York: Springer.

Byrne, B. M., Shavelson, R. J., \& Muthén, B. (1989). Testing for the equivalence of factor covariance and mean structures: The issue of partial measurement invariance. Psychological Bulletin, 105, 456-466.

Clogg, C. C. (1988). Latent class models for measuring. In R. Langeheine \& J. Rost (Eds.), Latent trait and latent class models (pp. 173-205). New York: Plenum Press.

Clogg, C. C. (1995). Latent class models. In G. Arminger, C. C. Clogg, \& M. E. Sobel (Eds.), Handbook of statistical modeling for the social and behavioral sciences (pp. 311-359). New York: Plenum Press.

Clogg, C. C. \& Manning, W. D. (1996). Assessing reliability of categorical measurements using latent class models. In A. von Eye \& C. C. Clogg (Eds.), Categorical variables in developmental research: Methods of analysis (pp. 169-182). New York: Academic Press.

Clogg, C. C. \& Shihadeh, E. S. (1994). Statistical models for ordinal variables. Thousand Oaks, CA: Sage.

Cohen, J. (1960). A coefficient of agreement for nominal scales. Educational and Psychological Measurement, 20, 37-46.

Cohen, J. (1968). Weighted kappa: Nominal scale agreement with provision for scaled disagreement or partial credit. Psychological Bulletin, 70, 213-220. 
Congdon, P. J. \& McQueen, J. (2000). The stability of rater severity in large-scale assessment programs. Journal of Educational Measurement, 37, 163-178.

Darroch, J. N. \& McCloud, P. I. (1986). Category distinguishability and observer agreement. Australian Journal of Statistics, 28, 371-388.

Dayton, C. M. (1998). Latent class scaling analysis. Thousand Oaks, CA: Sage.

Dayton, C. M. \& Macready, G. B. (1988). Concomitant-variable latent-class models. Journal of the American Statistical Association, 83, 173-178.

DeCarlo, L. T. (1998). Signal detection theory and generalized linear models. Psychological Methods, 3, 186-205.

DeCarlo, L. T. (2001, July). Signal detection models as structural equation models and latent class models: Examples of mixture, latent class, and multivariate signal detection models. Paper presented at the 2001 meeting of the Society for Mathematical Psychology, Providence, RI. Available at http://www.columbia.edu/ $\sim \operatorname{ld} 208$.

DeCarlo, L. T. (2002). Signal detection theory with finite mixture distributions: Theoretical developments with applications to recognition memory. Psychological Review, 109, 710-721.

Dillon, W. R. \& Mulani, N. (1984). A probabilistic latent class model for assessing interjudge reliability. Multivariate Behavioral Research, 19, 438-458.

Fleiss, J. L. (1981). Statistical methods for rates and proportions ( $2^{\text {nd }}$ ed.). New York: John Wiley \& Sons.

Goodman, L. A. (1974). The analysis of systems of qualitative variables when some of the variables are unobservable. Part I - a modified latent structure approach. American Journal of Sociology, 79, 1179-1259.

Goodman, L. A. \& Kruskal, W. H. (1954). Measures of association for cross classifications. Journal of the American Statistical Association, 49, 732-764.

Green, D. M. \& Swets, J. A. (1988). Signal detection theory and psychophysics (Rev. Ed.). Los Altos, CA: Peninsula Publishing.

Hagenaars, J. A. (1993). Loglinear models with latent variables. Newbury Park, CA: Sage.

Hambleton, R. K. \& Swaminathan, H. (1985). Item response theory. Boston, MA: Kluwer-Nijhoff Publishing.

Heinen, T. (1996). Latent class and discrete latent trait models: Similarities and differences. Thousand Oaks, CA: Sage.

Henkelman, R. M., Kay, I., \& Bronskill, M. J. (1990). Receiver operating characteristic (ROC) analysis without truth. Medical Decision Making, 10, 24-29.

Hoskens, M. \& Wilson, M. (2001). Real-time feedback on rater drift in constructedresponse items: An example from the Golden State examination. Journal of Educational Measurement, 38, 121-145.

Landis, J. R. \& Koch, G. G. (1977). The measurement of observer agreement for categorical data. Biometrics, 33, 159-174.

Lin, T. H. \& Dayton, C. M. (1997). Model selection information criteria for non-nested latent class models. Journal of Educational and Behavioral Statistics, 22, 249-264.

Little, R. J. A. \& Rubin, D. B. (1987). Statistical analysis with missing data. New York: John Wiley \& Sons.

Lord, F. M. (1980). Applications of item response theory to practical testing problems. Hillsdale, NJ: Lawrence Erlbaum Associates.

Macmillan, N. A. \& Creelman, C. D. (1991). Detection theory: A user's guide. New York: Cambridge University Press. 


\section{DeCarlo}

Marsh, H. W., Hau, K., Balla, J. R., \& Grayson, D. (1998). Is more ever too much? The number of indicators per factor in confirmatory factor analysis. Multivariate Behavioral Research, 33, 181-200.

McCutcheon, A. L. (1987). Latent class analysis. Newbury Park, CA: Sage.

McLachlan, G. \& Peel, D. (2000). Finite mixture models. New York: John Wiley \& Sons.

Mellenberg, G. J. (1996). Measurement precision in test score and item response models. Psychological Methods, 1, 293-299.

Muthén, L. K. \& Muthén, B. O. (1998). Mplus user's guide. Los Angeles: Authors.

Qu, Y., Tan, M., \& Kutner, M. H. (1996). Random effects models in latent class analysis for evaluating accuracy of diagnostic tests. Biometrics, 52, 797-810.

Quellmalz, E. S. (1985). Essay examinations. In T. Husen \& T. N. Postlethwaite (Eds.), International encyclopedia of education: Research and studies (pp.1709-1714). New York: Pergamon Press.

Quinn, M. F. (1989). Relation of observer agreement to accuracy according to a tworeceiver signal detection model of diagnosis. Medical Decision Making, 9, 196-206.

Rost, J. \& Langeheine, R. (Eds.). (1997). Applications of latent trait and latent class models in the social sciences. New York: Waxmann Münster.

Samejima, F. (1969). Estimation of latent ability using a response pattern of graded scores. Psychometrika Monograph No. 17, 34.

Swets, J. A. (1996). Signal detection theory and ROC analysis in psychology and diagnostics: Collected papers. Hillsdale, NJ: Erlbaum.

Swets, J. A., Tanner, W. P., \& Birdsall, T. G. (1961). Decision processes in perception. Psychological Review, 68, 301-340.

Uebersax, J. S. (1988). Validity inferences from interobserver agreement. Psychological Bulletin, 104, 405-416.

Uebersax, J. S. (1993a). A latent trait finite mixture model for the analysis of rating agreement. Biometrics, 49, 823-835.

Uebersax, J. S. (1993b). LLCA: Located latent class analysis user's manual. Unpublished manuscript, Wake Forest University, Winston-Salem, North Carolina. Available at http://ourworld.compuserve.com/homepages/jsuebersax/papers.htm.

Uebersax, J. S. (1993c). Statistical modeling of expert ratings on medical treatment appropriateness. Journal of the American Statistical Association, 88, 421-427.

Uebersax, J. S. (1999). Probit latent class analysis with dichotomous or ordered category measures: Conditional independence/dependence models. Applied Psychological Measurement, 23, 283-297.

Van der Heijden, P. G. M., Dessens, J., \& Bockenholt, U. (1996). Estimating the concomitant-variable latent-class model with the EM algorithm. Journal of Educational and Behavioral Statistics, 21, 215-229.

Vermunt, J. K. (1997a). LEM: A general program for the analysis of categorical data. Tilburg University, Netherlands: Author. Available at http://www.uvt.nl/faculteiten/ fsw/organisatie/departementen/mto/software $2 . h t m l$.

Vermunt, J. K. (1997b). Log-linear models for event histories. Thousand Oaks, CA: Sage Publications Inc.

Vermunt, J. K. \& Magidson, J. (2000). Latent gold user's guide. Belmont, MA: Statistical Innovations.

Walter, S. D. \& Irwig, L. M. (1988). Estimates of test error rates, disease prevalence and relative risk from misclassified data: A review. Journal of Clinical Epidemiology, 41, 923-937. 
L. DeCarlo

Appendix A

Sample LEM and Mplus Programs

\section{LEM Program}

* Binary response example (see Dayton, 1998)

* Table 3.1, Pleural thickening data (* denotes comments )

lat 1

$\operatorname{man} 3$

$\operatorname{dim} 2222$

lab X A B C

$\bmod \mathrm{X}$
* there is 1 latent variable (X)

* there are three manifest variables $(A, B, C)$

* dimensions of the variables

* labels for the variables

* model statement for $\mathrm{p}(\mathrm{X})$

$\mathrm{A} \mid \mathrm{X} \operatorname{cum}(\mathrm{a}) \quad\{\operatorname{cov}(\mathrm{X}, 1)\} *$ model statements for $c \circ n$ d $i$ i $\circ n$ a $l$ probabilities

$B \mid X \operatorname{cum}(a) \quad\{\operatorname{cov}(X, 1)\} * \operatorname{cum}(a)$ gives a logit link, design for cov below

$C \mid X \operatorname{cum}(a) \quad\{\operatorname{cov}(X, 1)\}$ rec 8

reo

wse separam.txt

$\operatorname{des}\left[\begin{array}{llllll}0 & 1 & 0 & 1 & 0 & 1\end{array}\right]$

dat dayton98.txt
* there are 8 records in an external file

* the data include record counts

* write out the standard errors to "separam.txt"

* the design matrix, three dummy coded Xs

* the name of the data file 


\section{DeCarlo}

\section{Mplus Program}

TITLE: Ordinal response example, multiple sclerosis data (see Landis \& Koch, 1977). See Appendix B for some comments on how the model is parameterized. Note: the chisquare and likelihood ratio tests of model fit are incorrect. (and will be corrected in a future release). See Mplus discussion at www.statmodel.com under "Latent variable mixture modeling" then "Goodnessof-fit statistics for mixture models" (August 3, 2001 post).

! Comments follow exclamation points.

DATA: FILE IS C:\mplus\files\landis.txt;

! NOTE: if data are in tabular form, it must be written out as individual records.

! This can be done using statistical packages (SAS was used).

VARIABLE: NAMES ARE Y1 y2;

CLASSES = Class (2);

CATEGORICAL $=\mathrm{Y} 1-\mathrm{Y} 2$;

ANALYSIS : TYPE=MIXTURE; ESTIMATOR=ML ;

MODEL : $\%$ OVERALL $\%$

f1 by y1@1; f2 by y2@1;

! Factors are included above to allow for non-zero means below.

$$
\begin{array}{llllll}
{[\mathrm{y} 1 \$ 1 *-1]} & (1) ; & {[\mathrm{y} 1 \$ 2 * 0]} & (2) ; & {[\mathrm{y} 1 \$ 3 * 1]} & (3) ; \\
{[\mathrm{y} 2 \$ 1 *-1]} & (4) ; & {[\mathrm{y} 2 \$ 2 * 0]} & (5) ; & {[\mathrm{y} 2 \$ 3 * 1]} & (6) ;
\end{array}
$$

! The numbers in parentheses restrict the thresholds to be equal across the latent classes. \%class\#1\%

[f1@0 f2@0];

\% Class\# $2 \%$

$\left[\begin{array}{lll}f 1 * 1 & f 2 * 1]\end{array}\right.$

! The above statement allows the means to be nonzero in one latent class.

! This gives the detection parameters. OUTPUT: sampstat techl; 


\section{Appendix $B$}

\section{Some Notes on using LEM and Mplus}

When fitting latent class SDT models with LEM, it should be recognized that there are two equivalent solutions, because it is arbitrary which latent class is used as the reference. If the values of $d_{j}$ are negative (and responses are coded so that larger numbers indicate higher confidence), then the thresholds give $c_{j k}, x_{1}^{*}=0$ corresponds to the lower latent class, and $x_{2}^{*}=1$ corresponds to the higher class. If the values of $d_{j}$ are positive, then $c_{j k}$ equals the threshold value plus $d_{j}, x_{2}^{*}=0$ is high and $x_{1}^{*}=1$ is low; note that the standard errors of $c_{j k}$ are then computed as the square root of the following: the variance of the given threshold plus the variance of $d_{j}$ plus two times the covariance of the threshold and $d_{j}$; these quantities can be obtained using the wse command, as shown in Appendix A.

For both LEM and Mplus, it is also important to check that the solution does not represent a local maxima by running the program several times with different starting values. If a larger value of the log-likelihood is obtained, then the solution with a smaller value is a local maxima, and the solution with larger value should be used.

For Mplus, the model is parameterized as a latent class model with two (or more) classes and the criteria (thresholds) are restricted to be equal across the classes. Means are included by using factors; the model is given by Equations 149-151 in the Mplus user's guide (1998). Note that, for the current version (2.01), the goodness of fit statistics are incorrect, but this will be corrected in a future release. 